\title{
Meine Zeit mit Prof. Wolfgang Hach
}

Am 1. Juli 1977 fing ich, einer der ersten Fachärzte für Gefäßchirurgie, als Oberarzt in der WILLIAM-HARVEY-KLINIK am Kaiserberg in Bad Nauheim an. Die Klinik mit privatem Träger war Anfang 1975 als Sanatorium eröffnet worden. Wolfgang Hach hatte als Chefarzt den Ausbau mit einem klinischen Trakt, einschließlich zweier Operationssäle und einer Überwachungsstation, zur Bedingung gemacht. Diese wurde nach eineinhalb Jahren erfüllt. So bestand die Klinik aus 80 „klinischen“ Betten und 190 „Kurbetten“ und einer stark frequentierten Ermächtigungsambulanz. Damit hatte Wolfgang Hach erstmals ein durchgehendes Behandlungskonzept Ambulanz-Klinik-Nachsorge verwirklicht. Wir waren ein funktionierendes Gefäßzentrum, lange bevor dieser Begriff zertifiziert wurde.

Ich konnte mich davon überzeugen, dass es gut funktionierte. Das Konzept wurde von vielen Kliniken 20 Jahre später übernommen und als innovativ und zukunftsträchtig propagiert. Die klinischen Anteile hatten die Bezeichnung „angiologisch-gefäßchirurgische Stationen“. Also keine Trennung von internistischen und chirurgischen Gefäßpatienten, sondern eine interdisziplinäre Diagnostik und Therapie. Auch dieses Konzept wurde viele Jahre später als effektivste Lösung gefeiert. Diese interdisziplinäre Idee war schon früh realisiert, weil Wolfgang Hach selbst interdisziplinär war: Er war sowohl Facharzt für Chirurgie als auch Facharzt für Innere Medizin. Im Rahmen seiner vielen Weiterbildungsstationen hatte er an der Radiologischen Klinik der Universität Frankfurt eine Schlüsseltechnik für seine folgenden wissenschaftlichen Leistungen entwickelt: die aszendierende Press-Phlebografie. Darauf basierend stellte er erstmalig die ganze Phlebologie auf eine wissenschaftliche Basis. In der Zeit davor wurden schon Operationen der Stammvarikose nach Babcock durchgeführt, aber keine chirurgische und auch gefäßchirurgische Klinik betrieb das mit großem Engagement, als letztes im Programm und oft den jüngsten Assistenten überlassen. Daneben gab es „Verödungspäpste“, die ein großes Einzugsgebiet beherrschten. Und die Patienten kamen alle wieder - also

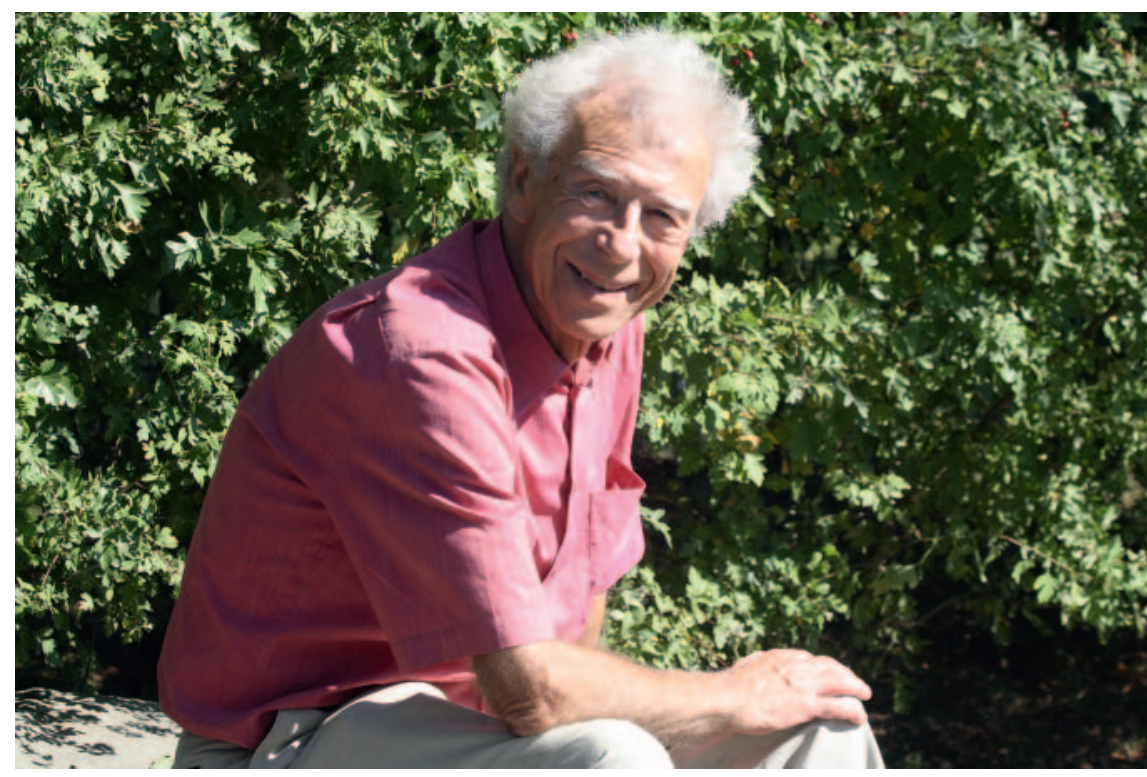

Professor Hach in seinem Garten. Quelle: Privatarchiv Familie Hach.

konnte die Methode doch nicht schlecht sein. Oder andere Einzelkämpfer arbeiteten in Praxen mit manchmal obskuren Methoden vor sich hin.

Mit der aszendierenden Press-Phlebografie konnten neben den tiefen Venen erstmals die Stammvenen und die insuffizienten transfaszialen Verbindungen dargestellt werden. Man sah bildlich vor sich, was zu tun ist, um eine erfolgreiche Operation durchzuführen. Es ließ sich die Stammvarikose in Stadien einteilen. Als Konsequenz daraus genügte eine Stadiengerechte Operation. In den meisten Fällen war eine Entfernung des Venenstamms bis unter das Knie ausreichend. So konnten intakte Anteile der Vene erhalten bleiben und vielleicht für später notwendige arterielle Rekonstruktionen und auch für Koronarbypässe genutzt werden.

Zudem ließen sich lästige neurologische Irritationen durch den dort eng benachbarten Nervus saphenus vermeiden. Selbst ein optimales Operationsergebnis kann durch Missempfindungen am Unterschenkel für den Patienten unbefriedigend sein.

Eine weitere Schlussfolgerung aus den unzähligen Phlebografien war die Beobachtung einer verstärkten Knickbildung der Vena poplitea bei langjährig bestehender, schwerer Stammvarikose. Es ließ sich eine Dilatation und Elongation der femoropoplitealen tiefen Venen wissenschaftlich dokumentieren. Die Klappen der tiefen Venen können bei entsprechender Dilatation nicht mehr suffizient schließen und so entsteht ein Reflux im tiefen Venensystem. Mit dem Namen „sekundäre femoropopliteale Insuffizienz“ wurde dieses Krankheitsbild von Wolfgang Hach treffend benannt. Die Ursache ist eine Überlastung der tiefen Venen durch das ständige Rezirkulationsvolumen. Eine dicke Varikose ist also nicht nur ein Schönheitsproblem, das von Männern oft unter den Hosen versteckt wird. Ob diese Spätfolgen nach Entfernung der Varizen reversibel sind, wurde leider nie konsequent untersucht. Schlussfolgerung ist, dass eine Varikose eher frühzeitig saniert werden sollte, bevor Spätschäden an den tiefen Venen manifest werden.

Die Formulierungskunst komplexer Krankheitsbegriffe zeigte sich bei Wolfgang Hach auch beim „arthrogenen Stauungssyndrom“. Es wurde beobachtet, dass auch bei intaktem Venensystem durch Einschränkung der Beweglichkeit, vor allem des oberen Sprunggelenks, eine venöse Stauung entsteht, die bis zum Ulcus cruris führen kann. Die Ursache ist der Ausfall der venösen Pumpfunktion durch die Gelenkbewegungen. Dieses 
Phänomen wurde im Rahmen einer Doktorarbeit systematisch untersucht. Nach den Erkenntnissen der Phlebografie war ein verbliebener Magnastumpf fast immer die Ursache einer klinisch relevanten Rezidiv-Varikose. Deswegen war bei der Operation die exakte Darstellung der Mündungsregion der Vena saphena magna und Ligatur mit nicht resorbierbaren Fäden von Anfang an Standard in der Klinik. Wenn ein Magnarezidiv operiert werden musste, machten wir die Inzision oberhalb oder lateral der alten Narbe, die meist unterhalb der Leistenfalte verlief, und es wurde erst die tiefe Vene dargestellt. An dieser entlang haben wir nach distal präpariert und eigentlich immer einen dicken Saphenastumpf gefunden, der dann entsprechend versorgt wurde. Wer diese Entwicklung mitgemacht hat, kann eigentlich die jetzt propagierte Unwichtigkeit der Saphenakrosse bei den endoskopischen Verfahren nicht verstehen.

Das weitere wissenschaftliche Interesse von Wolfgang Hach galt den Folgeerscheinungen der chronischen Venenkrankheiten. Cockett hatte nachweisen können, dass mit einem großen traumatisierenden Eingriff am Unterschenkel mit Beseitigung aller transfaszialen Verbindungen (die nach ihm benannten Cockett'schen Perforansvenen) varikös bedingte Unterschenkelgeschwüre geheilt werden konnten. Die ausgedehnten Operationswunden waren praktisch immer mit langwierigen Wundheilungsstörungen belastet.

Auf einer Reise nach Argentinien wurde Wolfgang Hach eine dort geläufige Operation vorgeführt. Von einer Inzision oberhalb der Hautveränderungen wurden mit einem Spatel subfaszial alle Perforansvenen blind durchtrennt. Der folgende Blutschwall kam nach Hochhalten des Beins und Kompression zum Stehen.

Wolfgang Hach hat diese heroische Methode an unserer Klinik erfolgreich übernommen. Erst als Prof. Hauer die subfasziale endoskopische Perforansdissektion entwickelt hatte, haben wir diese Methode im größeren Stil praktiziert. Unter Sicht konnte jetzt die Perforansdissektion erfolgen, was einem Chirurgen immer lieber ist als das brutal aussehende blinde Vorgehen mit dem Spatel. Dabei zeigte sich, dass die Muskulatur der darunterliegenden Kompartimente deutliche Zeichen einer schlechten Durchblutung aufwies. In systematischen Untersuchungen zur Faszienstruktur konnte festgestellt werden, dass diese verändert war. Elektronenmikroskopische Befunde von Prof. Staubesand konnten das nachweisen. Die Struktur der Faszien war chaotisch verändert und dadurch die Elastizität verloren gegangen. Die chronische Venenstauung hatte also nicht nur die Haut und Subkutis, sondern auch die Faszie betroffen. Vor allem beim Stehen konnten erhöhte Kompartmentdrucke gemessen werden. Auch computertomografisch waren deutliche Gewebsveränderungen nachweisbar. Die Konsequenz war eine Entlastung der Muskelkompartimente durch eine Faszienspaltung. Diese erfolgte unmittelbar paratibial, weil dadurch auch hier verlaufende Perforansvenen durchtrennt wurden. So wurde die endoskopisch subfasziale Perforansdissektion mit der paratibialen Fasziotomie kombiniert. Systeme zur subfaszialen endoskopischen Perforansdissektion werden von der Industrie angeboten. Ein Fasziotom dazu hat Wolfgang Hach selbst entwickelt. Die klinischen Erfolge waren sehr gut, die Kompartmentdrucke wurden messbar niedriger. Eine für die Evidenz notwendige Studie haben wir nicht gemacht, weil die Ausgangsbefunde so individuell unterschiedlich sind, dass keine vergleichbaren Kollektive möglich waren.

Auch für das chronisch-venöse Stauungssyndrom hat Wolfgang Hach eine Einteilung in 4 Stadien formuliert, einschließlich angepasster Therapieempfehlungen.

Er hatte die Fähigkeit, sein Denken zu strukturieren und auf der Basis gesicherter Erkenntnisse auch sperrige Sachverhalte mit eigens dafür geschaffenen Begriffen treffend zu formulieren. In Konsequenz zu dieser Entwicklung wollte Wolfgang Hach auch für die schwersten Krankheitsbilder eine Lösung finden, z. B. für die oft jahrzehntelang bestehenden zirkulären Ulzerationen, die alleine durch ihren Geruch und die aufwendige Versorgung alle sozialen Bindungen unmöglich machen. Solch ein Patient kam verzweifelt in unsere Klinik mit dem Wunsch, beide Unterschenkel zu amputieren, weil er sein langjähriges Leiden, das sein Leben zerstört hatte, beenden wollte. Wolfgang Hach hat es gewagt, die gesamten zirkulären Ulzera, einschließlich der Faszie, en bloc zu resezieren und die Wunden mit Mesh-graft-Transplantaten zu decken.
Die Abheilung war verzögert, aber schließlich erfolgreich, sodass der Eingriff am anderen Bein folgen konnte. Diese krurale Faszienresektion gehörte ab da zum Repertoire der Klinik, wenn sie auch nie wieder in diesem extremen Ausmaß notwendig wurde.

Die Phlebologie wurde durch das Lebenswerk von Wolfgang Hach zu einer ernstzunehmenden, logisch fundierten Wissenschaft entwickelt, die von anderen Disziplinen als gleichwertig anerkannt wurde. Davon droht vieles wieder verloren zu gehen. Die Notwendigkeit einer stationären Behandlung wird von den Krankenkassen nur noch selten anerkannt. Es gibt kaum mehr Radiologen, die die „invasive“ aszendierende Press-Phlebografie beherrschen. Diagnostik wird nur noch mit Duplex betrieben und leider in sehr unterschiedlichen Qualitäten. Als ich noch in der Klinik tätig war, habe ich grundsätzlich alle „auswärtigen“ Duplexbefunde angezweifelt, leider oft zu Recht. Die Phlebologie ist wieder den Einzelkämpfern überlassen, die ihre eigene Diagnose und anschließende endovaskuläre Therapie in eigener Regie betreiben. Industriegesponsert wird die Behandlung mit den endovaskulären Verfahren verharmlost und bagatellisiert. Viele Disziplinen sind beteiligt und an den Behandlungsort werden keine hohen Ansprüche gestellt. So sind auch verschiedene Standorte möglich, um die Amortisation der Geräte zu optimieren. Theoretisch ist das auch in einem Container auf Jahrmärkten möglich. Damit schließt sich der Kreis.

1984 wurde ich Leitender Arzt einer eigenen Gefäßchirurgie und mir wurde die Ambulanz übertragen. Außer getrennten Privatstationen verlief der Klinikalltag wie bisher interdisziplinär weiter. Auch wenn die Interessen von Wolfgang Hach mehr der Phlebologie galten, entwickelte sich die Gefäßchirurgie immer auf dem Level der Zeit. Er hat insbesondere die angiografischen Besonderheiten der diabetischen Angiopathie beschrieben. Intraarterielle Infusionen wurden als eine der wenigen Möglichkeiten der konservativen Angiologie bei vielen Patienten segensreich eingesetzt. Auch Amputationen wurden von den Gefäßchirurgen selbst gemacht. Von der Wiedereinführung der Kniegelenksexartikulation als Alternative zur Oberschenkelamputation profitierten 
viele Patienten mit einer wesentlich besseren Mobilität.

Neben der Kliniktätigkeit führte Wolfgang Hach eine angiologische Praxis im Herzen von Frankfurt/Main. Von dort wurden auch viele Klinikpatienten rekrutiert.

Am Nachmittag verließ er die Klinik in Richtung Praxis, mit einer großen Tasche, schwer von Röntgenbildern, die er dann noch spätabends befundete. Das änderte sich erst, als 1984 eine eigenständige Röntgenabteilung etabliert wurde.

Trotz dieses gut gefüllten Arbeitstages hat Prof. Hach nicht die Mühen gescheut, Symposien sowie regionale und auch internationale Kongresse in großer Zahl zu organisieren. Der Höhepunkt war der internationale Kongress der Deutschen Gesellschaft für Phlebologie, der als erster in der gerade neu aufgebauten „Alten Oper" in Frankfurt stattfand. Das Tanzparkett am Abend war noch sehr glatt.

Unzählige Publikationen hat Wolfgang Hach verfasst und zahlreiche Bücher „nebenher" geschrieben, in denen er seine Gedanken, Methoden und Erfolge darstellte. Er hat auch die jüngeren Mitarbeiter immer zu wissenschaftlicher Tätigkeit animiert und sie zu „freiwilligen“ Vorträgen auf seinen Kongressen eingespannt. Dabei konnten sie auf seine ungeheure Diasammlung zurückgreifen oder neue erstellen, die aber einer unerbittlichen Kontrolle unterlagen. Nur beste inhaltliche und grafische Qualität durfte die Klinik repräsentieren.

Trotz all der Hektik in seinem Alltag war er bei allem sehr exakt, fast penibel, aber auch fantasievoll, sogar mutig und innovativ und immer verantwortungsvoll; alles Eigenschaften, die einen guten Chirurgen ausmachen. Das kann ich nach meinen vielfältigen Erfahrungen beurteilen. Auch sein internistisches Wissen war für mich als Chirurg bewundernswert, obwohl ich durch die interdisziplinäre Tätigkeit selbst fast zum Angiologen geworden bin. Die vertrauensvolle Zusammenarbeit mit Wolfgang Hach war in all den gemeinsamen Jahren für mich ein Glücksfall. Der Umgang war immer fair, offen und vertrauensvoll. In diesem Punkt war Wolfgang Hach eher „Internist“, wenn ich an manche chirurgischen Chefs meiner Ausbildungszeit denke, die sich emotional und verhaltensmäßig nicht weit von offenbar militärischen Vorbildern entfernt hatten. Eine Harmonie von 2 Ärzten ist eher ungewöhnlich, aber auch nur bei gegenseitiger Wertschätzung möglich. Das trägt dazu bei, ein gutes Betriebsklima zu schaffen und eine leistungsstarke, engagierte Mannschaft zu schmieden, in der der gute „Harvey-Geist“ entstehen kann.

Die 20 gemeinsamen Jahre in der William-Harvey-Klinik waren eine Erfolgsgeschichte. Der Bekanntheitsgrad und der gute Ruf verbreiteten sich in ganz Deutschland und über die Grenzen hinaus. Die Zahl der Mitarbeiter wuchs von Jahr zu Jahr und die Operationsfrequenz hat sich verdreifacht. Wolfgang Hach hat sich 1980 an der Hautklinik der Universität Gießen habilitiert und erhielt 2 Jahre später die Professur. Kurz nach seinem 65. Geburtstag hat Wolfgang Hach die WilliamHarvey-Klinik verlassen, um in den „Unruhestand“ zu gehen. Es blieben ihm seine Frankfurter Praxis, Kongress- und Vortragsverpflichtungen und das Bücherschreiben. Mangels Patientenhintergrund hat er sich bei seinen Vorträgen zuneh- mend medizinhistorischen Themen zugewandt und aus dem Schatz seiner antiquarischen Bücher geschöpft. Zu seinen Ehren haben wir in Zusammenarbeit mit der Hautklinik Darmstadt im Dezember seines letzten Klinikjahres einen Kongress im Hotel Interkontinental in Frankfurt ausgerichtet. „Venenchirurgie live“ wurde von über 1000 Teilnehmern besucht. Ich bezeichne ihn rückblickend gerne als „Woodstock der Venenchirurgie“, weil es den Höhepunkt der Venenchirurgie darstellte mit Liveübertragungen aus den Operationssälen von Darmstadt und Bad Nauheim.

Die Tätigkeit in der Praxis hat Wolfgang Hach im letzten Jahr aufgegeben und ganz in die Hände seiner Tochter Viola gelegt. Die medizinischen Vorträge für die „Universität des dritten Lebensalters“ der Universität Frankfurt hat er weiterhin vor vielen 100 Zuhörern gehalten.

Zum 60. Geburtstag von Wolfgang Hach hatten wir schon mal eine Vortragsveranstaltung organisiert mit dem Titel „Phlebologie“, um die von ihm hergestellte Verbindung von Logik und Phlebologie hervorzuheben. Der folgende Festabend wurde nicht wie üblich mit einem Walzer, sondern mit einem Rock'n'Roll eröffnet. Eine Fake-Tina-Turner besang den Jubilar mit „You are simply the best“. Ich habe dem nichts hinzuzufügen.

\section{Dr. Gerhard Salzmann}

Bibliografie

DOI https://doi.org/10.1055/a-1148-3466 Phlebologie 2020; 49: 127-129

(c) Georg Thieme Verlag KG, Stuttgart · New York ISSN 0939-978X 